Pacific Journal of Mathematics

ANGULAR LIMITS OF LOCALLY FINITELY VALENT
HOLOMORPHIC FUNCTIONS 


\title{
ANGULAR LIMITS OF LOCALLY FINITELY VALENT HOLOMORPHIC FUNCTIONS
}

\author{
DAVID C. HADDAD
}

\begin{abstract}
A function $f$ defined in a domain $D$ is $n$-valent in $D$ if $f(z)-w_{0}$ has at most $n$ zeros in $D$ for each complex number $w_{0}$. The purpose of this paper is to show that a sufficient condition for a holomorphic function $f$ in $|z|<1$ to have angular limits almost everywhere on $|z|=1$ is that there exist a positive integer $n$ and a positive number $r_{0}$ such that $f$ is $n$-valent in each component of the set $\left\{z:|f(z)|>r_{0}\right\}$.
\end{abstract}

We have previously shown that the same conditions on $f$ imply that $f$ is a quasi-normal function of order at most $n-1[3$, Theorem 2], and $f$ has angular limits at a dense subset of $|z|=1$ [3, Corollary 1]. Note that the bound $n$ on the valence of $f$ is the same for each component of $\left\{z:|f(z)|>r_{0}\right\}$. This uniformity on $n$ is essential to the conclusion that $f$ has angular limits almost everywhere on $|z|=1$; for we have shown in the example in [2] that if the uniformity is dropped, then $f$ need not even have asymptotic values at a dense subset of $|z|=1$.

If $w=f(z)$ is a nonconstant, holomorphic function in $|z|<1$, we denote by $F$ the Riemann surface of $f^{-1}$ (as a covering surface over the $w$-plane). If $S$ is a subset of $|z|=1$, then $m(S)$ denotes the Lebesgue measure of $S$.

A Jordan arc $T=\{z=h(t): 0<t<1\}$ lying in a domain $D$ is a crosscut of $D$ if $h(t) \rightarrow z_{0} \in \partial D$ as $t \downarrow 0, h(t) \rightarrow z_{1} \in \partial D$ as $t \uparrow 1$, and $z_{0} \neq z_{1}$. If $z_{0}=z_{1}$, then $T$ is a loopcut of $D$.

If a holomorphic function $f$ in $|z|<1$ is $n$-valent in a component $D(r)$ of the set $\{z:|f(z)|>r\}$ then the connectivity of $D(r)$ is as most $n+1$ [3, Lemma 3]. We denote by $D^{*}(r)$ the simply connected domain obtained by adding to $D(r)$ those (at most $n$ ) components of $\{z:|f(z)| \leqq r\}$ that punch holes in $D(r)$.

LEMMA 1. Let $f$ be a nonconstant, holomorphic function in $|z|<1$ that is n-valent in each component of the set $\left\{z:|f(z)|>r_{0}\right\}$. For each $r>r_{0}$, let $\left\{D_{k}(r)\right\}$ denote the at most countable collection of components of $\{z:|f(z)|>r\}$. Then there exists a countable subset $E$ of $\left(r_{0}, \infty\right)$ such that $\partial D_{k}^{*}(r)$ is a Jordan curve for all $k$ and all $r \in\left(r_{0}, \infty\right)-E$.

Proof. Define a set $R=\left\{r: r>r_{0}\right.$, and $F$ has no branch points lying over the circle $|w|=r\}$. Then the set $\left(r_{0}, \infty\right)-R$ is at most countable. If $r \in R$, then for each $k, \partial D_{k}^{*}(r) \cap\{|z|<1\}$ consists of at 
most countably many crosscuts and loopcuts $T_{j}^{k}$ of $|z|<1$ by [2, Corollary 1].

We show that if for a fixed $k$ there are infinitely many curves $T_{j}^{k}$, then their diameters tend to zero as $j \rightarrow \infty$. If the diameters did not tend to zero, then the sequence $\left\{T_{j}^{k}\right\}$ would have an accumulation continuum in $|z| \leqq 1$. Since $f$ is a nonconstant, holomorphic function, $\left\{T_{j}^{k}\right\}$ cannot have an accumulation continuum in $|z|<1$. By [2, Theorem 3], $f$ has asymptotic values at a dense subset of $|z|=1$, and hence, by a theorem of MacLane [4, Theorem 1], the sequence $\left\{T_{j}^{k}\right\}$ of level curves cannot have an arc of $|z|=1$ for an accumulation continuum. Hence, the diameters of the curves $T_{j}^{k}$ tend to zero as $j \rightarrow \infty$.

We still must show that there exists a countable subset $E$ of $\left(r_{0}, \infty\right)$ such that $\partial D_{k}^{*}(r)$ has no double points for all $k$ and all $r \in$ $\left(r_{0}, \infty\right)-E$. Suppose to the contrary that $S$ is an uncountable subset of $R$ and that for each $r \in S$ there exists a component $D(r)$ of the set $\left\{z:|f(z)|>r_{0}\right\}$ such that $\partial D^{*}(r)$ has double points. This implies that for each $r \in S, \partial D^{*}(r)$ contains a loopcut $T_{r}$, since the curves comprising $D^{*}(r) \cap\{|z|<1\}$ are Jordan arcs for all $r \in R$. The domain $D^{*}(r)$ cannot be interior to a loopcut; for if it were, $f$ would be unbounded in $D^{*}(r)$ by the extended maximum principle, and, consequently, the locpcut would determine two distinct asymptotic tracts ending at one point contradicting [2, Theorem 2]. (See [4] or [2] for the definition of an asymptotic tract.) Let $G_{r}$ denote the domain interior to the loopcut $T_{r}$. The uncountable collection of open sets $G_{r}$ must contain a pair that intersect, say $G_{q}$ and $G_{s}$ where $q<s$. Since the loopcuts $T_{q}$ and $T_{s}$ cannot intersect inside $|\boldsymbol{z}|<1$, then $G_{q} \subset G_{s}$, and $T_{q}$ and $T_{s}$ end at the same point of $|z|=1$. By [2, Corollary 1], $T_{q}$ and $T_{s}$ determine at least two (since $q \neq s$ ) asymptotic tracts ending at one point contradicting [2, Theorem 2]. Thus, there must exist a countable subset $E$ of $(r, \infty)$ such that $\partial D_{k}^{*}(r)$ is a Jordan curve for all $k$ and all $r \in\left(r_{0}, \infty\right)-E$.

LEMMA 2. Let $f$ be a nonconstant, holomorphic function in $|z|<1$ that is n-valent in each component of $\left\{z:|f(z)|>r_{0}\right\}$. If $r_{1}>r_{0}$ and $D\left(r_{1}\right)$ is a component of $\left\{z:|f(z)|>r_{1}\right\}$, then $f$ has angular limits almost everywhere on $E\left(r_{1}\right)=\overline{D\left(r_{1}\right)} \cap\{|\boldsymbol{z}|=1\}$.

Proof. We assume $m\left(E\left(r_{1}\right)\right)>0$, for, otherwise, there is nothing to prove. For $r<r_{1}$, we denote by $D(r)$ the component of $\{z:|f(z)|>$ $r\}$ containing $D\left(r_{1}\right)$, and we write $E(r)=\overline{D(r)} \cap\{|\boldsymbol{z}|=1\}$. We first show that there exists $s \in\left(r_{0}, r_{1}\right)$ such that $\partial D^{*}(s)$ is a rectifiable Jordan curve.

By Lemma 1, the set $R=\left\{r \in\left(r_{0}, r_{1}\right)\right.$ such that $F$ has no branch 
points over $|w|=r$ and $\partial D^{*}(r)$ is a Jordan curve $\}$ is the whole interval $\left(r_{0}, r_{1}\right)$ minus possibly a set of measure zero. Let $C(r)=\partial D(r) \cap\{|z|<$ $1\}$, and let $\Gamma$ be the family $\{C(r): r \in R\}$. By passing to the Riemann surface $F$, it is not hard to show that the extremal length of the family $\Gamma$ is bounded by $2 n \pi \log r_{1} / r_{0}$, and this implies $\partial D(r)$ is rectifiable for infinitely many values $r \in R$ (for example, see [2, Theorem 1]). Thus, we can choose $s \in\left(r_{0}, r_{1}\right)$ such that $\partial D^{*}(s)$ is a rectifiable Jordan curve.

By the Riemann mapping theorem and Carathéodory's theorem on boundary correspondence there exists a homeomorphism $g$ of $\overline{D^{*}(s)}$ onto $|\zeta| \leqq 1$ that is a conformal mapping of $D^{*}(s)$ onto $|\zeta|<1$. Since the connectivity of $D(s)$ is finite, $\left|f\left(g^{-1}(\zeta)\right)\right|>s$ in some annulus $t<|\zeta|<1$. Hence, $f \circ g^{-1}$ has angular limits almost everywhere on $|\zeta|=1$ by a simple extension of theorems of Fatou [1, p. 19] and F. and M. Riesz $\left[1\right.$, p. 22] on angular limits. Since $\partial D^{*}(s)$ is a rectifiable Jordan curve, $g^{-1}$ maps a set of measure zero on $|\zeta|=1$ onto a set of measure zero on $\partial D^{*}(s)$ by a theorem of F. and M. Riesz [1, p. 50]. Thus $f$ has asymptotic values almost everywhere on $E(s)$ and hence angular limits almost everywhere on $E(s)$ by [3, Theorem 3]. This completes the proof of the lemma since $E(s) \supset E\left(r_{1}\right)$.

LEMMA 3. Let $\left\{I_{j}\right\}$ be a sequence of mutually disjoint open arcs on $|\boldsymbol{z}|=1$, and let $C=\bigcup_{j} I_{j}$. Let $f$ be a continuous function on $\{|z|<1\} \cup C$ that is holomorphic in $|z|<1$. Let $|f(z)|=r_{0}$ for $z \in$ $C,|f(0)|>r_{0}$, and the set $D=\left\{z:|z|<1,|f(z)|>r_{0}\right\}$ be a connected set whose boundary contains the circle $|\boldsymbol{z}|=1$. If $f$ is n-valent in $D$, then $|f(0)| \leqq r_{0} \exp \left[2 \pi^{4} n / m(C)^{2}\right]$.

Proof. Let $\gamma(r)$ be the level set $\{z:|f(z)|=r\}$. The proof consists of finding bounds on the extremal length $\lambda(\Gamma)$ of the family $\Gamma=$ $\left\{\gamma(r): r_{0}<r<|f(0)|\right.$, and $F$ has no branch points lying over $\left.|w|=r\right\}$. By passing to the Riemann surface $F$, it can be shown that $\lambda(\Gamma) \leqq$ $2 \pi n / \log |f(0)| / r_{0}$ (for example, see [2, Theorem 1]).

By our hypotheses on $f$, each arc $I_{j}$ must be separated from the point $z=0$ by a level curve of $\{z:|f(z)|=r\}$ for each $r$ in the interval $\left(r_{0},|f(0)|\right)$. None of these curves can be relatively compact curves encircling the point $z=0$ by the maximum principle. Thus, the Euclidean length of a level curve separating $I_{j}$ from $z=0$ is bounded below by $\min \left(2,(2 / \pi) m\left(I_{j}\right)\right)$. Hence, the Euclidean length of each $\gamma(r) \in \Gamma$ is bounded below by $(1 / \pi) m(C)$. By considering the linear density $\rho(z)$ defined to be 1 on $D$ and 0 elsewhere, we can easily obtain the inequality $\lambda(\Gamma) \geqq\left(1 / \pi^{3}\right) m(C)^{2}$. Combining the two bounds on $\lambda(\Gamma)$ we have $|f(0)|<r_{0} \exp \left[2 \pi^{4} n / m(C)^{2}\right]$, which completes the proof of the lemma. 
A point $e^{i \theta}$ is a Plessner point for a function $f$ defined in $|z|<$ 1 if for every Stolz angle $S$ at $e^{i \theta}$, the cluster set of $f$ at $e^{i \theta}$ with respect to the domain $S$ is total.

THEOREM. A sufficient condition for a holomorphic function $f$ in $|z|<1$ to have finite angular limits almost everywhere on $|\boldsymbol{z}|=1$ is that there exist a positive number $r_{0}$ and a positive integer $n$ such that $f$ is n-valent in each component of the set $\left\{z:|f(z)|>r_{0}\right\}$.

Proof. Suppose to the contrary that the set of points of $|z|=1$ at which $f$ does not have finite angular limits has positive measure. Then, by a theorem of Plessner [1, p. 147] and a theorem of Priwalow $[1$, p. 146], $f$ must be a nonconstant function whose set of Plessner points $P$ has positive measure.

For each $r>0$, let $\left\{D_{j}(r)\right\}$ denote the at most countable collection of components of the set $\{z:|f(z)|>r\}$. By Lemma 1, there exists $r_{1}>r_{0}$ such that $\partial D_{j}^{*}\left(r_{1}\right)$ is a Jordan curve for each $j$ and $F$ has no branch points over the circle $|w|=r_{1}$. Thus, $\partial D_{j}^{*}\left(r_{1}\right) \cap\{|z|<1\}$ consists of at most countably many level curves which are crosscuts of $|z|<1$. Write $D_{j}=D_{j}\left(r_{1}\right), E_{j}=\overline{D_{j}^{*}} \cap\{|z|=1\}$, and $E_{j}^{\prime}=\{|z|=1\}-$ $E_{j}$. Since by Lemma $2, f$ has angular limits almost everywhere on $\mathrm{U}_{j} E_{j}$, we can assume $P \subset \bigcap_{j} E_{j}^{\prime}$. Let $\omega_{j}$ be the harmonic measure in $D_{j}^{*}$ of the set $\partial D_{j}^{*} \cap\{|z|<1\}$. We need the following lemma whose proof we postpone.

Lemma 4. There exists a harmonic function $v$ in $|z|<1$ having angular limit 0 almost everywhere on $\bigcap_{j} E_{j}^{\prime}$, and $\omega_{j}(z) \geqq 1-v(z)$ for $z \in D_{j}^{*}(j=1,2, \cdots)$.

Thus, there exists a point $z_{0} \in P$ at which $v$ has angular limit 0 . Then, by the definition of $P$, there exists a sequence $\left\{z_{k}\right\}$ of points lying inside a Stolz angle at $z_{0}$ and converging to $z_{0}$ such that $\left|f\left(z_{k}\right)\right|>$ $r_{1}$ for each $k$ and $f\left(z_{k}\right) \rightarrow \infty$ as $k \rightarrow \infty$. At most finitely many $z_{k}$ can lie in the same component $D_{j}$ since $z_{0} \in P \subset \bigcap_{j} E_{j}^{\prime}$. Hence, we can assume (by taking subsequences if necessary) that $z_{j} \in D_{j}(j=1,2, \cdots)$ and $D_{j} \cap D_{k}=\phi$ for $j \neq k$. By Lemma $4, \omega_{j}\left(z_{j}\right) \rightarrow 1$ as $j \rightarrow \infty$.

By the Riemann mapping theorem and Carathéodory's theorem on boundary correspondence, there exists a homeomorphism $g_{j}$ of $\overline{D_{j}^{*}}$ onto $|\zeta|<1$ that is a holomorphic map of $D_{j}^{*}$ onto $|\zeta|<1$ sending $z_{j}$ into 0 . Applying Lemma 3 to the function $h_{j}=f \circ g^{-1}$ and the set $C_{j}=$ $g_{j}\left(\partial D_{j}^{*} \cap|z|<1\right)$ we have $\left|h_{j}(0)\right| \leqq r_{1} \exp \left[2 \pi^{4} n / m\left(C_{j}\right)^{2}\right]$. On the one hand, $h_{j}(0)=f\left(z_{j}\right) \rightarrow \infty$ as $j \rightarrow \infty$. On the other hand, $m\left(C_{j}\right)=$ $2 \pi \omega_{j}\left(g_{j}^{-1}(0)\right)=2 \pi \omega_{j}\left(z_{j}\right) \rightarrow 2 \pi$ as $j \rightarrow \infty$, and this implies $h_{j}(0) \nrightarrow \infty$ as $j \rightarrow \infty$. Thus to complete the proof of the theorem, we need only 
prove Lemma 4.

Let $u_{j}$ be the harmonic measure in $|z|<1$ of the set $E_{j}^{\prime}$, and let $v_{k}(z)=\sum_{j=1}^{k}\left(1-u_{j}(z)\right)$. Clearly, $\left\{v_{k}\right\}$ is an increasing sequence of nonnegative harmonic functions, and $v_{k}$ has angular limit 0 at each point of the set $\bigcap_{j=1}^{k} E_{j}^{\prime}$. Since the set $\overline{D_{j}^{*}} \cap \overline{D_{q}^{*}}$ can contain at most two points for $j \neq q$, each point $e^{i \theta}$ lies in at most one of the sets $E_{1}, E_{2}, \cdots, E_{k}$ for all but finitely many values of $\theta$ in the interval $[0,2 \pi)$. Hence, $\overline{\lim }_{z \rightarrow e^{i \theta}} v_{k}(z) \leqq 1(k=1,2, \cdots)$ for all but finitely many values $\theta \in[0,2 \pi)$. It follows from the extended maximum principle that $v_{k}(z) \leqq 1$ for $|z| \leqq 1(k=1,2, \cdots)$. By Harnack's theorem, the sequence $\left\{v_{k}\right\}$ converges in $|z|<1$ to a bounded harmonic function $v(z)$. Let $I=\left\{\theta: 0 \leqq \theta<2 \pi, e^{i \theta} \varepsilon \bigcap_{j} E_{j}^{\prime}\right.$, and $v, v_{1}, v_{2}, \cdots$ have angular limits at $\left.e^{i \theta}\right\}$. Then, writing $v\left(e^{i \theta}\right)$ for the angular limit of $v$ at $e^{i \theta}$, we have

$$
\begin{aligned}
\int_{I} v\left(e^{i \theta}\right) d \theta & =\int_{I} v\left(e^{i \theta}\right)-v_{k}\left(e^{i \theta}\right) d \theta \\
& \leqq \int_{0}^{2 \pi} v\left(e^{i \theta}\right)-v_{k}\left(e^{i \theta}\right) d \theta \\
& =v(0)-v_{k}(0)
\end{aligned}
$$

Thus, $v$ has angular limit 0 at $e^{i \theta}$ for almost all $\theta \in I$, since $v(0)-$ $v_{k}(0) \rightarrow 0$ as $k \rightarrow \infty$. Since the set $\bigcap_{j} E_{j}^{\prime}-\left\{e^{i \theta}: \theta \in I\right\}$ has measure zero by Fatou's theorem, $v$ has angular limit 0 almost everywhere on $\bigcap_{j} E_{j}^{\prime}$. Clearly, $v(z) \geqq v_{j}(z) \geqq 1-u_{j}(z)$ for all $j$ and $|z|<1$, and by Carleman's principle of domain extension, $\omega_{j}(z) \geqq u_{j}(z)$ for $z \in D_{j}^{*}(j=$ $1,2, \cdots)$. This completes the proof of Lemma 4 and hence of the theorem.

REMARK. The conclusion of the theorem raises the following question. Are all functions that satisfy the hypotheses of the theorem of bounded characteristic? This seems to be a difficult question to answer. The best we can presently show is that $T(r)=o(1 / 1-r)$, where $T$ is the Nevanlinna characteristic of $f$.

\section{REFERENCES}

1. E. F. Collingwood and A. J. Lohwater, The Theory of Cluster Sets, Cambridge University Press, 1966.

2. D. C. Haddad, Asymptotic values of finitely valent functions, Duke Math. J., 39 (1972), 391-367.

3. - Boundary behavior and quasi-normality of finitely valent holomorphic functions, to appear, Canad. J. Math., (1973).

4. G. R. MacLane, Asymptotic Values of Holomorphic Functions, Rice University Studies, 49 No. 1 (1963). 
Received June 22, 1972. The author is indebted to the late Professor G. R. MacLane for his considerable assistance.

West Virginia College of Graduate Studies 


\section{PACIFIC JOURNAL OF MATHEMATICS}

\section{EDITORS}

RICHARD ARENS (Managing Editor)

University of California

Los Angeles, California 90024

R. A. Beaumont

University of Washington

Seattle, Washington 98105
J. DUGUNDJI*

Department of Mathematics

University of Southern California

Los Angeles, California 90007

D. Gilbarg and J. Milgram

Stanford University

Stanford, California 94305

\section{ASSOCIATE EDITORS}
E. F. BECKENBACH
B. H. NeumanN
F. WOLF
K. YosHIDA

\section{SUPPORTING INSTITUTIONS}

UNIVERSITY OF BRITISH COLUMBIA
CALIFORNIA INSTITUTE OF TECHNOLOGY
UNIVERSITY OF CALIFORNIA
MONTANA STATE UNIVERSITY
UNIVERSITY OF NEVADA
NEW MEXICO STATE UNIVERSITY
OREGON STATE UNIVERSITY
UNIVERSITY OF OREGON
OSAKA UNIVERSITY

UNIVERSITY OF BRITISH COLUMBIA

UNIVERSITY OF CALIFORNIA

MONTANA STATE UNIVERSITY

UNIVERSITY OF NEVADA

OREGON STATE UNIVERSITY

OSAKA UNIVERSITY
UNIVERSITY OF SOUTHERN CALIFORNIA

STANFORD UNIVERSITY

UNIVERSITY OF TOKYO

UNIVERSITY OF UTAH

WASHINGTON STATE UNIVERSITY

UNIVERSITY OF WASHINGTON

\section{AMERICAN MATHEMATICAL SOCIETY} NAVAL WEAPONS CENTER

* C. R. DePrima California Institute of Technology, Pasadena, CA 91109, will replace J. Dugundji until August 1974. 


\section{Pacific Journal of Mathematics}

\section{Vol. 48, No. $1 \quad$ March, 1973}

Jan Aarts and David John Lutzer, Pseudo-completeness and the product of Baire

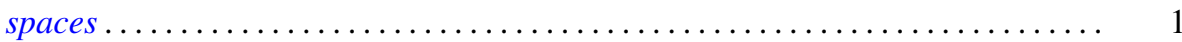

Gordon Owen Berg, Metric characterizations of Euclidean spaces ............ 11

Ajit Kaur Chilana, The space of bounded sequences with the mixed topology ..... . 29

Philip Throop Church and James Timourian, Differentiable open maps of

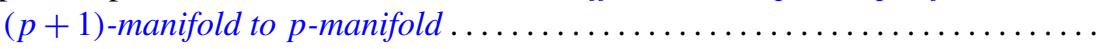

P. D. T. A. Elliott, On additive functions whose limiting distributions possess a finite

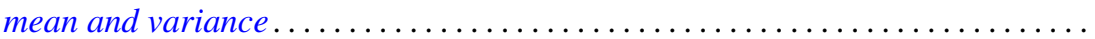

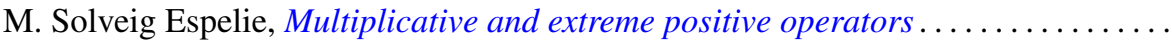

Jacques A. Ferland, Domains of negativity and application to generalized convexity

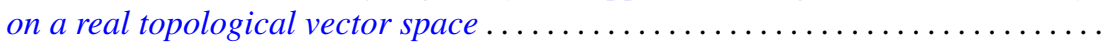

Michael Benton Freeman and Reese Harvey, A compact set that is locally holomorphically convex but not holomorphically convex ...............

Roe William Goodman, Positive-definite distributions and intertwining

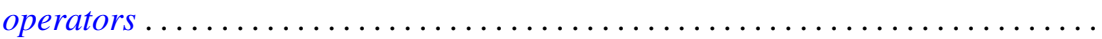

Elliot Charles Gootman, The type of some $C^{*}$ and $W^{*}$-algebras associated with

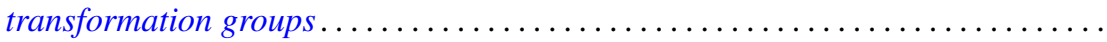

David Charles Haddad, Angular limits of locally finitely valent holomorphic

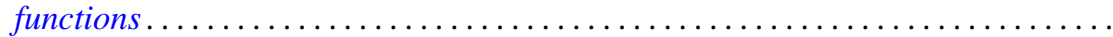

William Buhmann Johnson, On quasi-complements .

William M. Kantor, On 2-transitive collineation groups of finite projective spaces...

Joachim Lambek and Gerhard O. Michler, Completions and classical localizations of right Noetherian rings

Kenneth Lamar Lange, Borel sets of probability measures ......

David Lowell Lovelady, Product integrals for an ordinary differential equation in a Banach space

Jorge Martinez, A hom-functor for lattice-ordered groups .........

W. K. Mason, Weakly almost periodic homeomorphisms of the two sphere ....

Anthony G. Mucci, Limits for martingale-like sequences .......

Eugene Michael Norris, Relationally induced semigroups ...

Arthur E. Olson, A comparison of c-density and $k$-density ......

Donald Steven Passman, On the semisimplicity of group rings of linear groups.

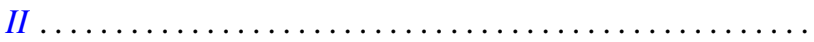

Charles Radin, Ergodicity in von Neumann algebras .

P. Rosenthal, On the singularities of the function generated by the Bergman operator of the second kind.

Arthur Argyle Sagle and J. R. Schumi, Multiplications on homogeneous spaces,

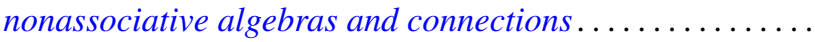

Leo Sario and Cecilia Wang, Existence of Dirichlet finite biharmonic functions on

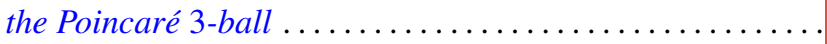

Ramachandran Subramanian, On a generalization of martingales due to Blake ..

Bui An Ton, On strongly nonlinear elliptic variational inequalities.

Seth Warner, A topological characterization of complete, discretely valued

fields. 